\title{
Analysis of geomagnetic disturbances dynamics during increased solar activity and magnetic storms (according to the measurements of INTERMAGNET station network)
}

\author{
Oksana Mandrikova ${ }^{1, *}$, Anastasia Rodomanskay ${ }^{1}$, and Alexander Zaitsev ${ }^{2}$ \\ ${ }^{1}$ Institute of Cosmophysical Research and Radio Wave Propagation FEB RAS, 684034 Paratunka, \\ Kamchatka region, Russia \\ ${ }^{2}$ Pushkov Institute of Terrestrial Magnetism, Ionosphere and Radio Waves Propagation of the Russian \\ Academy of Sciences, 108840 Troitsk, Moscow, Russian
}

\begin{abstract}
We present and describe an automated method for analysis of magnetic data and for detection of geomagnetic disturbances based on wavelet transformation. The parameters of the computational algorithms allow us to estimate the characteristics of non-uniformly scaled peculiar properties in the variations of geomagnetic field that arise during increasing geomagnetic activity. The analysis of geomagnetic data before and during magnetic storms was carried out on the basis of the method according to ground station network. Periods of increasing geomagnetic activity, which precede and accompany magnetic storms, are highlighted. The dynamic of geomagnetic field variation in the auroral zone is considered in detail.
\end{abstract}

\section{Introduction}

Analysis of geomagnetic data and the study of the processes in the magnetosphere provide valuable information about the state of electromagnetic fields in near-Earth space [1]. The paper presents the results of data processing and analysis from a geomagnetic station network in Russia, equatorial station (resource used: http://www.intermagnet.org) and stations of the auroral zone (resource used: http://www.intermagnet.org, http://www.geomag.nrcan.gc.ca/index-en.php, http://cygnus.raunvis.hi.is// halo/lrv.html ).

The study was based on the use of wavelet transform methods [2, 3]. This mathematical apparatus is one of the most effective modern methods of complex structure data analysis [4-6]. Geomagnetic data have an irregular structure and contain local features of different amplitudes and durations, which contain important information about the processes in the magnetosphere. To distinguish and to identify them is a difficult task [4-7]. Traditional methods of data analysis based on smoothing procedures and spectral analysis methods cannot investigate in detail the fast-changing structure of geomagnetic field variations and

*Corresponding author: oksanam1@ mail.ru 
lead to the loss of significant information. The wavelet transform allows us to study shortperiod oscillations of the geomagnetic field and to evaluate their parameters [8-10]. In this paper, we used algorithms developed on the basis of wavelet transform for analysis of geomagnetic data and extracted different-scale perturbations of the geomagnetic field [11]. The data processing results confirmed the possibility of the occurrence of short-term increases in geomagnetic activity at equatorial latitudes during periods of small variations in the interplanetary magnetic field (IMF) and an increase in auroral activity. The dynamics of geomagnetic field variation in the auroral zone is considered in detail. The connection of the distinguished disturbances with IMF parameters and AE-index is shown. We have experimentally confirmed the assumption that the critical feature of stability in the magnetosphere is the result of a combination of interplanetary medium state and internal magnetospheric activity.

\section{Description of the method}

Based on the wavelet transform, the time series of geomagnetic data $f(t)$ is presented as a combination of functions $[8,10]$ :

$$
f(t)=\sum_{n} c_{m, n} \varphi_{m, n}(t)+\sum_{j \in I} d_{j, n} \Psi_{j, n}(t)+e(t)
$$

where $\phi_{m}=\left\{\phi_{m, n}\right\}_{n \in Z}$ is the basis of a smoothing scaling function; $\Psi_{j}=\left\{\Psi_{j, n}\right\}_{n \in Z}$ is the wavelet basis; coefficients $c_{m, n}=\left\langle f, \phi_{m, n}\right\rangle$ and $d_{j, n}=\left\langle f, \Psi_{j, n}\right\rangle ; m$ are the scale level of decomposition; $I$ is a set of indices; $j$ is a set of indices; component $e(t)$ is noise. Identification of model (1) components is described in [10].

As a measure of magnetic disturbance on a scale $j$, following the works of $[8,10]$, we used the quantity:

$$
A_{j}=\max _{n}\left(\left|d_{j, n}\right|\right) .
$$

Then geomagnetic disturbances (define a set of coefficients $\left\{d_{j, n}\right\}_{j \in I}$ in (1)) can be identified on the basis of threshold functions $F_{1}$ and $F_{2}$ [10]:

$$
\begin{aligned}
& f(t)=f_{\text {trend }}(t)+\sum_{j_{\text {pert }}, n} F_{1}\left(d_{j_{\text {pert }}, n}\right) \Psi_{j_{\text {pert }}, n}(t)+\sum_{j_{\text {pert }}, n} F_{2}\left(d_{j_{\text {pert }}, n}\right) \Psi_{j_{\text {pert }}, n}(t)+e(t), \\
& F_{1}(x)=\left\{\begin{array}{c}
0, \text { if }|x| \leq T_{j_{p e r t}, 1} \text { or }|x|>T_{j_{p e r t}, 2} \\
x, \text { if } T_{j_{p e r t}, 1}<|x| \leq T_{j_{p e r t}, 2}
\end{array} ;\right. \\
& F_{2}(x)=\left\{\begin{array}{l}
0, \text { if }|x| \leq T_{j_{p e r t}, 2} \\
x, \text { if } \quad|x|>T_{j_{p e r t}, 2}
\end{array},\right.
\end{aligned}
$$

The threshold $T_{j_{\text {cosiny }}, 1}$ determines infirm geomagnetic disturbances, and the threshold $T_{j_{\text {сохими }}, 2}$ determines strong geomagnetic disturbances.

Taking into account the equivalence of discrete and continuous wavelet expansions [2, 3 ], for more detailed information about the properties of the analyzed function $f$, we used a continuous wavelet transform:

$$
\left(W_{\psi} f\right)(b, a):=|a|^{-1 / 2} \int_{-\infty}^{\infty} f(t) \Psi\left(\frac{t-b}{a}\right) d t, \Psi-\text { wavelet }, f \in L^{2}(R), a, b \in R, a \neq 0 \text {. }
$$


Then, in accordance with (2), a measure of geomagnetic disturbances intensity on scale $a$ at time $t=b$ is the amplitude of wavelet coefficients:

$$
v_{b, a}=\left|\left(W_{\psi} f\right)(b, a)\right| .
$$

Then the field perturbation intensity at time $t=b$ can be estimated based on the value:

$$
I_{b}=\sum_{a}\left|\left(W_{\psi} f\right)(b, a)\right|
$$

Taking into account the nonstationarity of geomagnetic data and following the relation (3), adaptive thresholds were used to extract positive and negative perturbations of the geomagnetic field [10]:

$$
F_{T_{a}}\left[\left(W_{\psi} f\right)(b, a)\right]=\left\{\begin{array}{c}
\left(W_{\psi} f\right)(b, a), \text { если }\left(W_{\psi} f\right)(b, a) \geq T_{a} \\
0, \text { если }\left|\left(W_{\psi} f\right)(b, a)\right|<T_{a} \\
-\left(W_{\psi} f\right)(b, a), \text { если }\left(W_{\psi} f\right)(b, a)<-T_{a}
\end{array}\right.
$$

where $T_{a}=U \times S t_{a}$ is threshold function, $S t_{a}^{l}=\sqrt{\frac{1}{l-1} \sum_{k=1}^{l}\left(\left(W_{\psi} f\right)(b, a)-\overline{\left(W_{\psi} f\right)(b, a)}\right)^{2}}$, $\overline{\left(W_{\psi} f\right)(b, a)}$ is the average value of continuous wavelet transform coefficients, calculated in a sliding time window of length $l, U$ is the threshold coefficient. The window length $l$ determines the size of the sliding time window, within which we evaluate the perturbations. The threshold coefficient $U$ determines the geomagnetic disturbance intensity.

Similarly to (5) the intensity of short-period perturbations at time $t=b$ was estimated on the basis of:

$$
E_{b}=\sum_{a} F_{T_{a}}\left[\left(W_{\psi} f\right)(b, a)\right]
$$

Then the intensity of positive and negative perturbations at time $t=b$ can be estimated as:

$$
E_{b}^{ \pm}=\sum_{a} F_{T_{a}}\left[\left(W_{\psi} f\right)(b, a)^{ \pm}\right]
$$

\section{Experimental results and discussion}

In this paper, we process the geomagnetic data with minute resolution from the Russian stations «Yakutsk» YAK, «Paratunka» PET, equatorial station «Guam» GUA, USA, and stations of the auroral zone «Abisko» ABK, Sweden, «Barrow » BRW, USA, «College» CMO, USA, «Fort Churchill» FCC, Canada, «Narsarsuaq» NAQ, Greenland, «Yellowknife» YKC, Canada, «Sanikiluaq» SNK, Canada, and «Leirvogur» LVR, Iceland (see Table 1 and Figure 1). We used magnetic data obtained at the observatories in accordance with the INTERMAGNET standards (www.inrtermagnet.org), that is, data in which there is no noise, jumps, and long-term artificial and anthropogenic effects. The results of our analysis were compared with the data of interplanetary magnetic field and solar wind parameters (https://omniweb.gsfc.nasa.gov/ow.html). In order to analyze geomagnetic activity in the auroral zone, we used the geomagnetic activity indices AE, AU, $\mathrm{AO}$ and $\mathrm{AL}$ (http://isgi.unistra.fr/index.php) [12]. The values of $\mathrm{AU}$ and $\mathrm{AL}$ indices characterize the intensity of the eastern (afternoon sector) and western (morning sector) polar electrojet currents in the auroral region $[12,13]$. The measure of the total maximum amplitude of the electrojet currents in the East and West directions is the AE-index [12, 13]. The AO-index determines the displacement of the midpoint AU and AL from a quiet level 
at a certain time and is an approximate measure equivalent to the zonal current, regardless of whether the current is in the ionosphere or in the magnetosphere [12]. In order to analyze the equatorial current system, we used the Dst-index (http://isgi.unistra.fr) [14].

Table 1. Observatories the data of which were used

\begin{tabular}{|l|c|c|c|c|c|c|}
\hline \multicolumn{1}{|c|}{ Observatory } & $\begin{array}{c}\text { IAGA } \\
\text { code }\end{array}$ & $\begin{array}{c}\text { Geographi } \\
\text { cal } \\
\text { latitude }\end{array}$ & $\begin{array}{c}\text { Geographical } \\
\text { longitude }\end{array}$ & $\begin{array}{c}\text { Geomagne } \\
\text { tic latitude }\end{array}$ & $\begin{array}{c}\text { Geomagnetic } \\
\text { longitude }\end{array}$ & $\begin{array}{c}\text { Local time } \\
\text { (LT) }\end{array}$ \\
\hline Barrow & BRW & $71^{0} 19.5^{\prime} \mathrm{N}$ & $156^{0} 37.2^{\prime} \mathrm{W}$ & $70^{0} 06.0^{\prime} \mathrm{N}$ & $110^{0} 34,2 \mathrm{~W}$ & UTC-8 \\
\hline College & CMO & $64^{0} 52.2^{\prime} \mathrm{N}$ & $147^{0} 51.5^{\prime} \mathrm{W}$ & $68^{0} 33.6^{\prime} \mathrm{N}$ & $57^{0} 13,2 \mathrm{~W}$ & UTC-8 \\
\hline Yellowknife & YKC & $62^{0} 28.8^{\prime} \mathrm{N}$ & $114^{0} 28.8^{\prime} \mathrm{W}$ & $67^{0} 12.0^{\prime} \mathrm{N}$ & $28^{0} 51,0 \mathrm{~W}$ & UTC-6 \\
\hline Fort Churchill & FCC & $58^{0} 45.5^{\prime} \mathrm{N}$ & $9^{0} 05.3^{\prime} \mathrm{W}$ & $67^{0} 12.0^{\prime} \mathrm{N}$ & $28^{0} 51,0 \mathrm{~W}$ & UTC-5 \\
\hline Sanikiluaq & SNK & $56^{0} 30.0^{\prime} \mathrm{N}$ & $79^{0} 12.0^{\prime} \mathrm{W}$ & $65^{0} 43.2^{\prime} \mathrm{N}$ & $08^{0} 17,4 \mathrm{~W}$ & UTC-4 \\
\hline Narsarsuaq & NAQ & $61^{0} 11.7^{\prime} \mathrm{N}$ & $45^{0} 25.0^{\prime} \mathrm{W}$ & $69^{0} 00.0^{\prime} \mathrm{N}$ & $38^{0} 49.2^{\prime} \mathrm{W}$ & UTC-2 \\
\hline Leirvogur & LVR & $64^{0} 10.8^{\prime} \mathrm{N}$ & $21^{0} 42.0^{\prime} \mathrm{W}$ & $68^{0} 42.6^{\prime} \mathrm{N}$ & $70^{0} 24.0^{\prime} \mathrm{W}$ & UTC+0 \\
\hline Abisko & ABK & $68^{0} 21.7^{\prime} \mathrm{N}$ & $18^{0} 43.4^{\prime} \mathrm{E}$ & $66^{0} 04.8^{\prime} \mathrm{N}$ & $113^{0} 53.4^{\prime} \mathrm{E}$ & UTC+2 \\
\hline Yakutsk & YAK & $61^{0} 57.6^{\prime} \mathrm{N}$ & $129^{0} 39.4^{\prime} \mathrm{E}$ & $52^{0} 54.0^{\prime} \mathrm{N}$ & $162^{0} 23.4^{\prime} \mathrm{E}$ & UTC+09 \\
\hline Guam & GUA & $13^{0} 35.4^{\prime} \mathrm{N}$ & $144^{0} 52.5^{\prime} \mathrm{E}$ & $5^{0} 58.8^{\prime} \mathrm{N}$ & $143^{0} 03.0^{\prime} \mathrm{E}$ & UTC+10 \\
\hline Paratunka & PET & $52^{0} 58.3^{\prime} \mathrm{N}$ & $158^{0} 15.0^{\prime} \mathrm{E}$ & $46^{0} 19.8^{\prime} \mathrm{N}$ & $136^{0} 52.2^{\prime} \mathrm{E}$ & UTC+12 \\
\hline
\end{tabular}

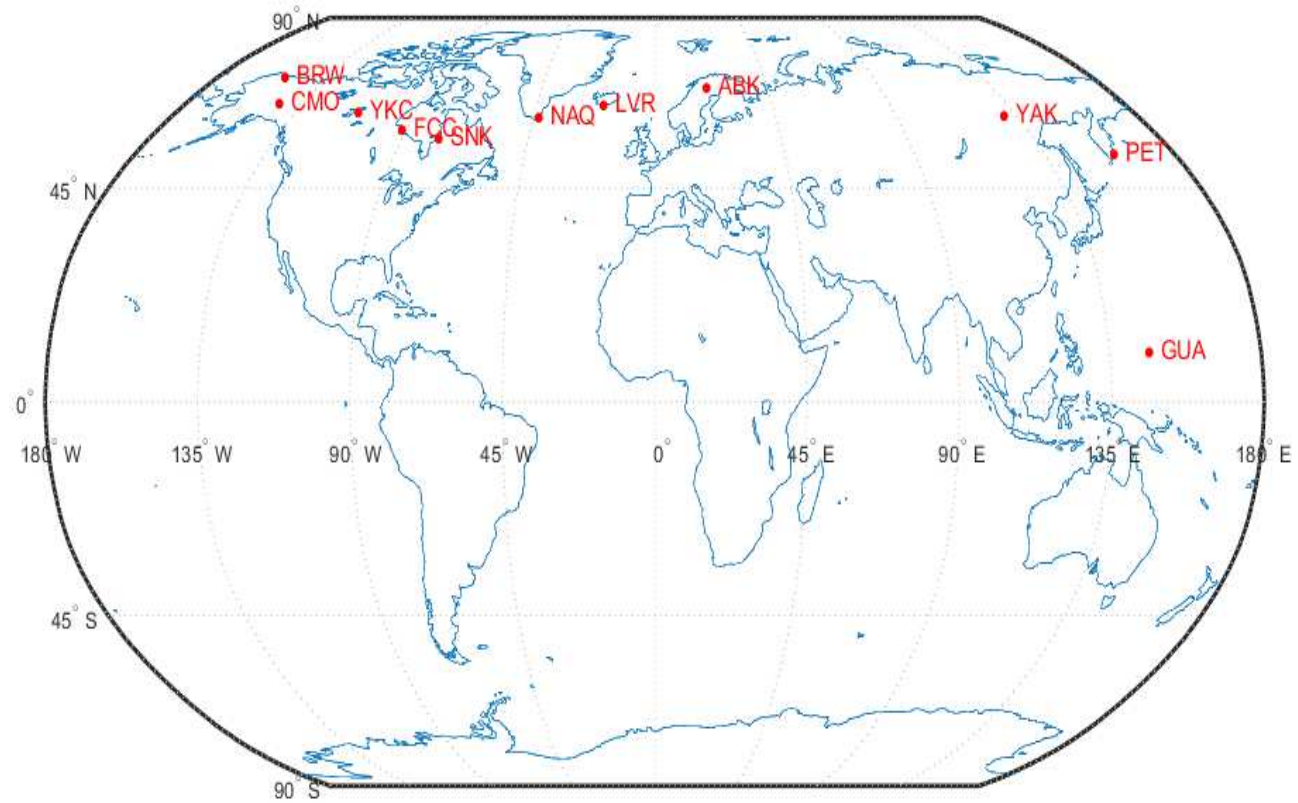

Fig. 1. Geographical position of observatories that provided data used in this study.

The first event analyzed on July 9, 2017 (see Figure 2-5), was caused by the arrival of an inhomogeneous accelerated flow arising from an extensive coronal hole (http://spaceweather.com). On the eve of the magnetic storm on July 08 from 10:30 to 15:00 UT during the periods of IMF Bz component oscillations (Fig.2c) within $+/-1.5 \mathrm{nT}$, there was a slight increase in auroral activity indices (Fig.2a) and short-term weak geomagnetic disturbances appeared at auroral stations BRW, YKC (Fig.5e). From 12:30 to 15:00 UT geomagnetic disturbances occurred at meridian stations YAK and PET (Fig.4e,f). In the evening from 19:30 till 22:20 UT, weak geomagnetic disturbances occurred at all 
analyzed stations (see Fig.2g,h and 4e,f). The selected effects are consistent with the results of the studies presented in [10] and confirm the possibility of short-term pre-storm anomalous changes in the dynamics of geomagnetic field variations at the background of small fluctuations in IMF Bz-component and slight increases in the auroral activity indices. It should also be noted that from 05:00 to 07:30 UT on July 08, during the period of positive values of IMF Bz component at the auroral stations BRW, CMO and FCC, an increase in geomagnetic activity is observed (Fig.5e), and with a delay of about 2 hours, synchronous short-term geomagnetic disturbances are detected at all meridian stations YAK, PET and GUA (Fig.4e,f). During the same period, a moderate increase in Dst-index is observed (see Fig. 2e), which is probably due to the manifestation of the current system of polar disturbances.

During the morning hours of July 09 around 00:10 UT, when the IMF Bz-component turned southward and reached $-10 \mathrm{nT}$ (Fig. 2c), there is an increase in the indices of auroral activity (see Fig. 2a) and an increase of geomagnetic activity at all analyzed stations (Fig.2g,h and 4e,f). With the arrival of a high-speed flow, the solar wind speed gradually increased from $350 \mathrm{~km} / \mathrm{s}$ to $420 \mathrm{~km} / \mathrm{s}$ (Fig. 2b), fluctuations of the IMF southern component increased to $\pm 12 \mathrm{nT}$. During the period of Bz-component increasing oscillations (Fig.4c), an increase in auroral activity indices (Fig. 4a) and the appearance of strong disturbances at YAK and PET stations are observed. Their intensity reached maximum values at YAK station (Fig. 4f). The results of processing the auroral station data (see. Fig. 3,5) show a correlation between the increases in the auroral activity indices and the fluctuations of IMF southern component (Fig. 3b,c) with the increase in geomagnetic disturbances at the stations in the morning and at night (Fig. 3e and 5e). This is probably due to an increase in the intensity of the western electrojet currents in the auroral region [15]. Detailed analysis of auroral zone data (see. Fig. 5e) allows one to determine the position of electrojet maxima in longitude, and show a picture of their interaction. 


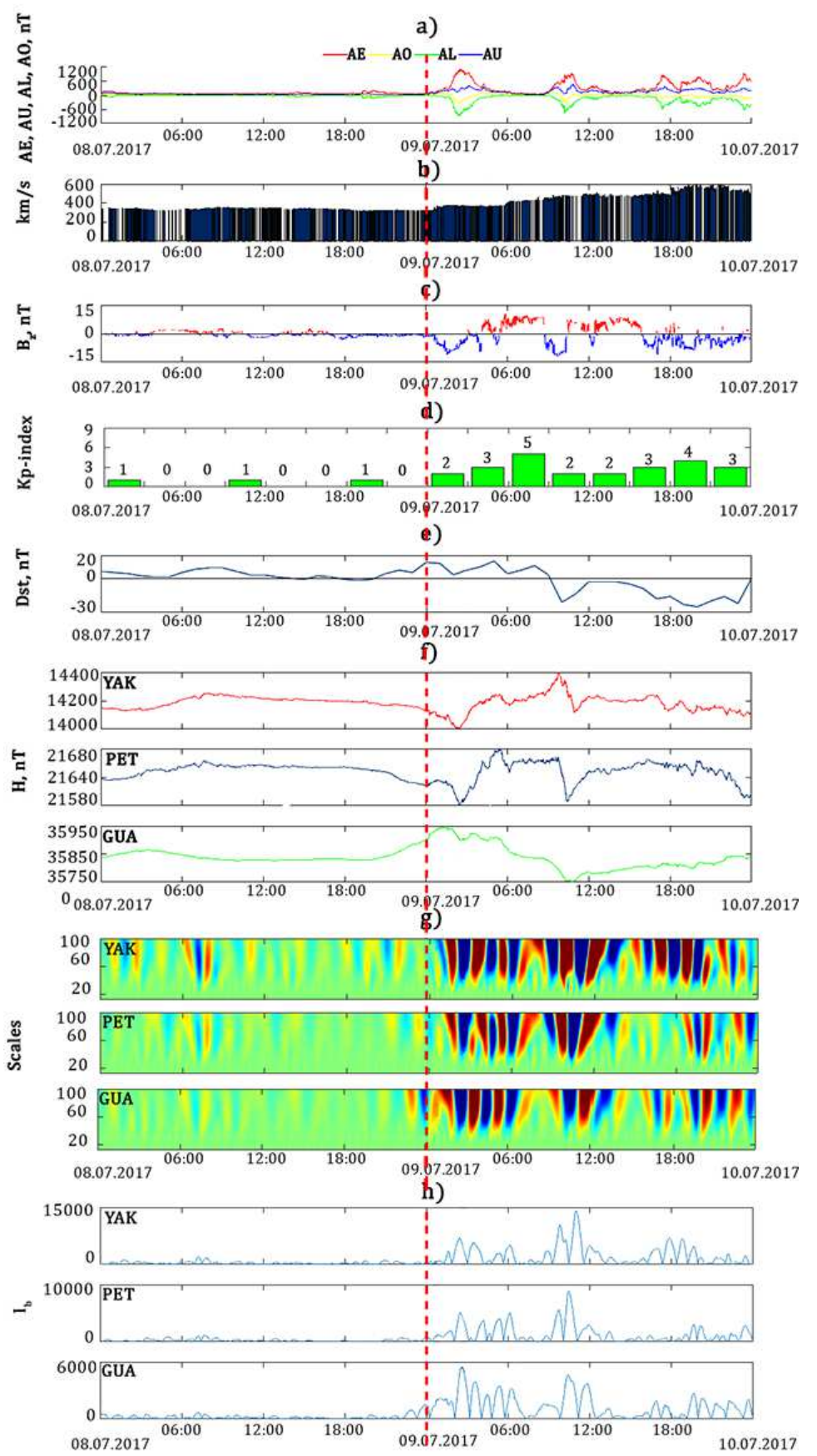

Fig. 2. Data processing results for the period from 08.07.2017 till 10.07.2017:

a) auroral indices $\mathrm{AE}, \mathrm{AO}, \mathrm{AL}$ and $\mathrm{AU}$; b) solar wind speed; c) Bz-component of interplanetary magnetic field, red color indicates positive values, blue - negative; d) Kp-index; e) Dst- index; f) H-component of the magnetic field at PET, YAK and GUA stations; g) wavelet spectrum of geomagnetic disturbances in the regions of PET, YAK and GUA stations (operation (4));

h) absolute values of disturbance intensity in the regions of PET, YAK and GUA stations (operation (5)). The vertical dashed line indicates the magnetic storm onset. 


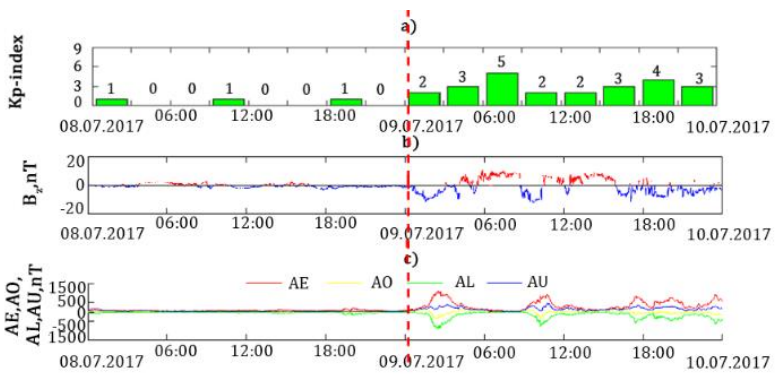

d)

Univerșal time

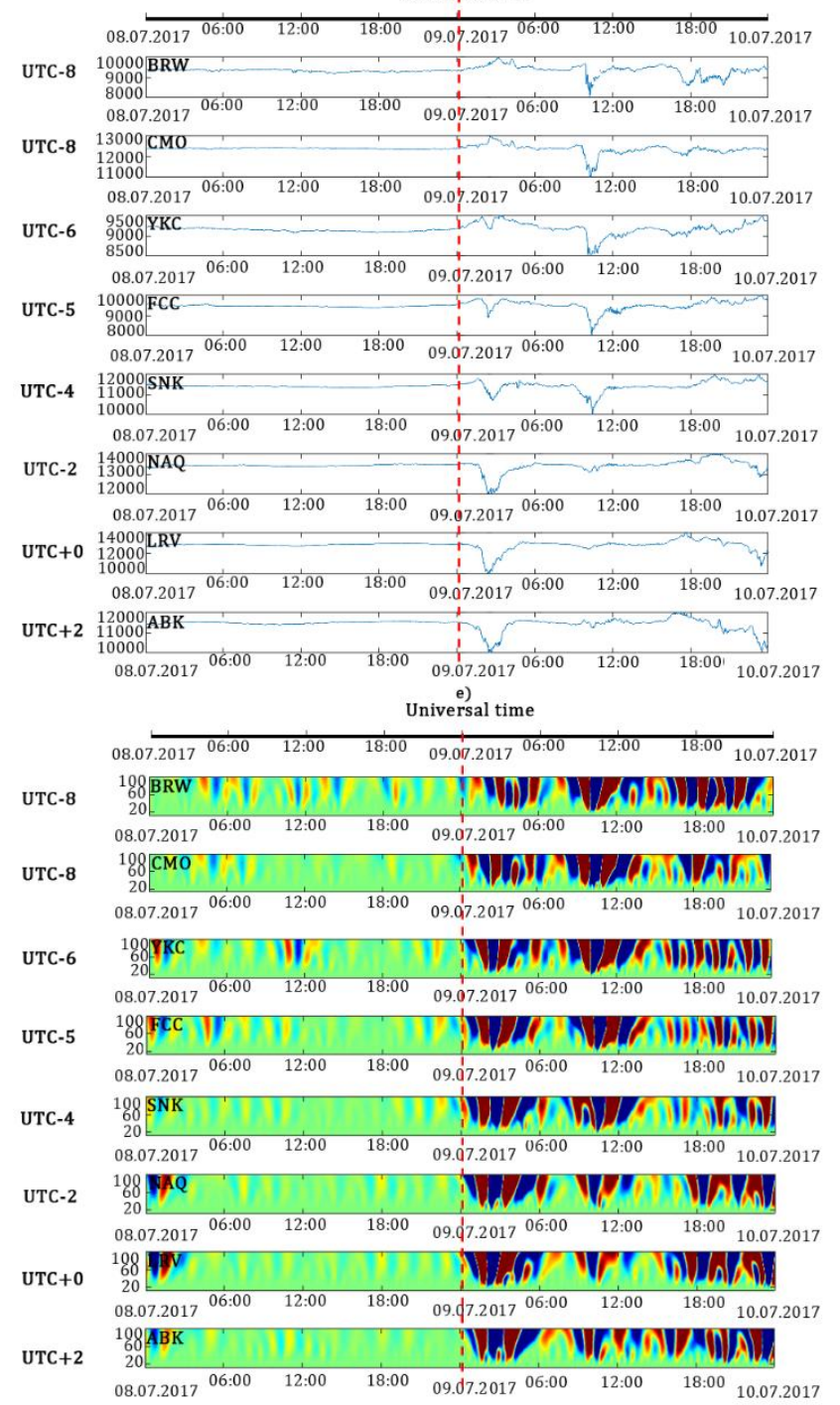

Fig. 3. Results of the auroral zone data processing for the period from 08.07.2017 till 10.07.2017:

a) Kp-index; b) Bz-component of interplanetary magnetic field, red color indicates positive values, blue - negative; c) auroral indices $\mathrm{AE}, \mathrm{AO}, \mathrm{AL}$ and $\mathrm{AU}$; d) $\mathrm{H}$-component of the magnetic field;

e) wavelet spectrum of geomagnetic disturbances in the areas of the auroral zone stations (operation (4)). The vertical dashed line indicates the magnetic storm onset. 


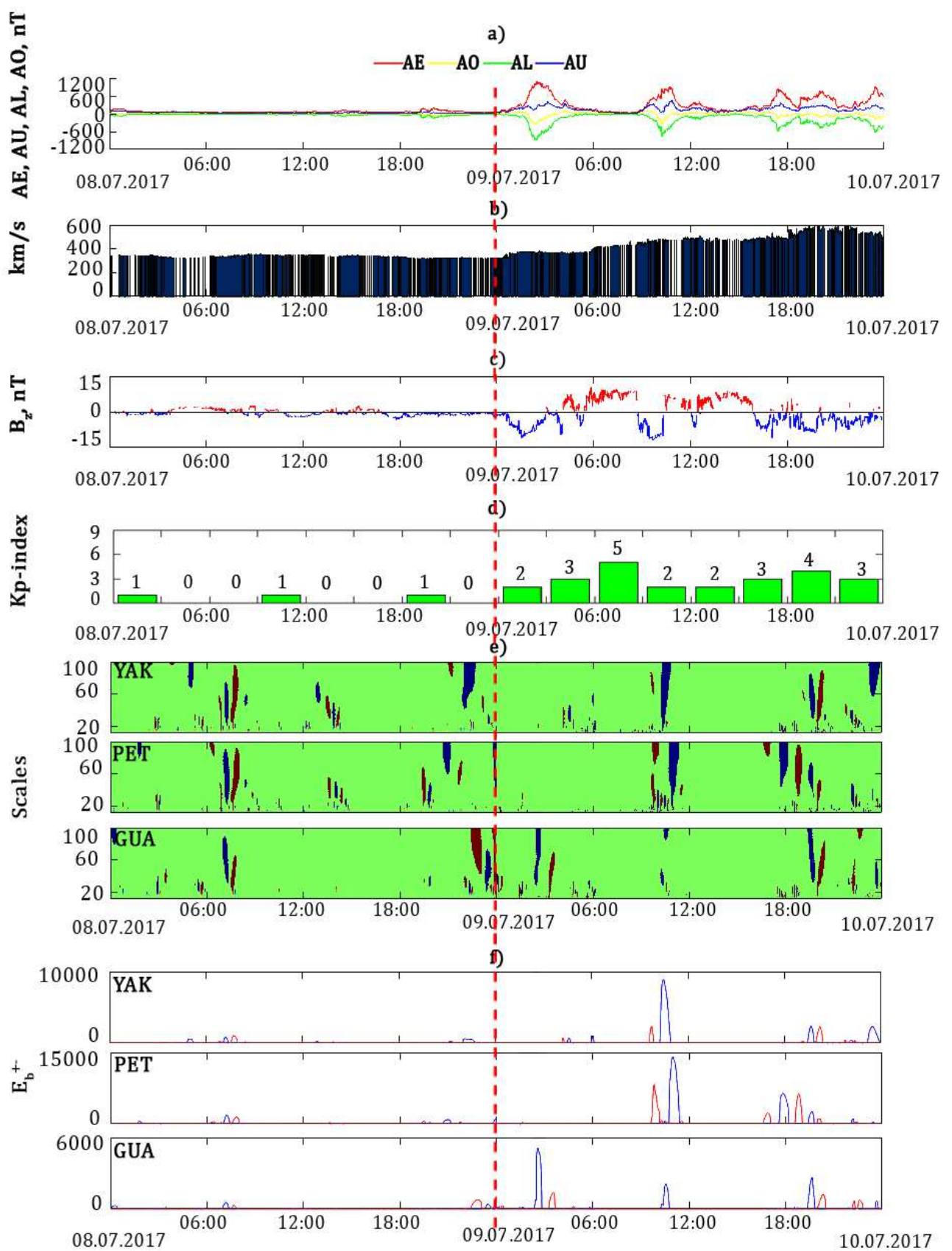

Fig. 4. Data processing results for the period from 08.07.2017 till 10.07.2017:

a) auroral indices $\mathrm{AE}, \mathrm{AO}, \mathrm{AL}$ and $\mathrm{AU}$; b) solar wind speed; c) Bz-component of interplanetary magnetic field, red color indicates positive values, blue - negative; d) $\mathrm{Kp}$-index; e) calculations according to (6), red color indicates positive perturbations, blue - negative ones; f) calculations according to (7), red color indicates positive perturbation, blue - negative ones. The vertical dashed line indicates the magnetic storm onset. 

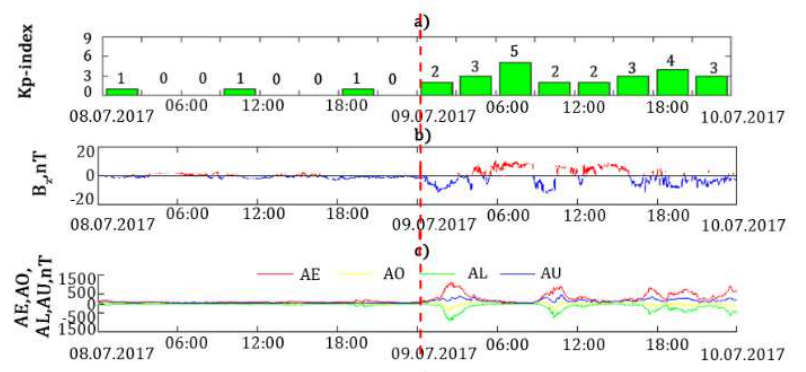

d)

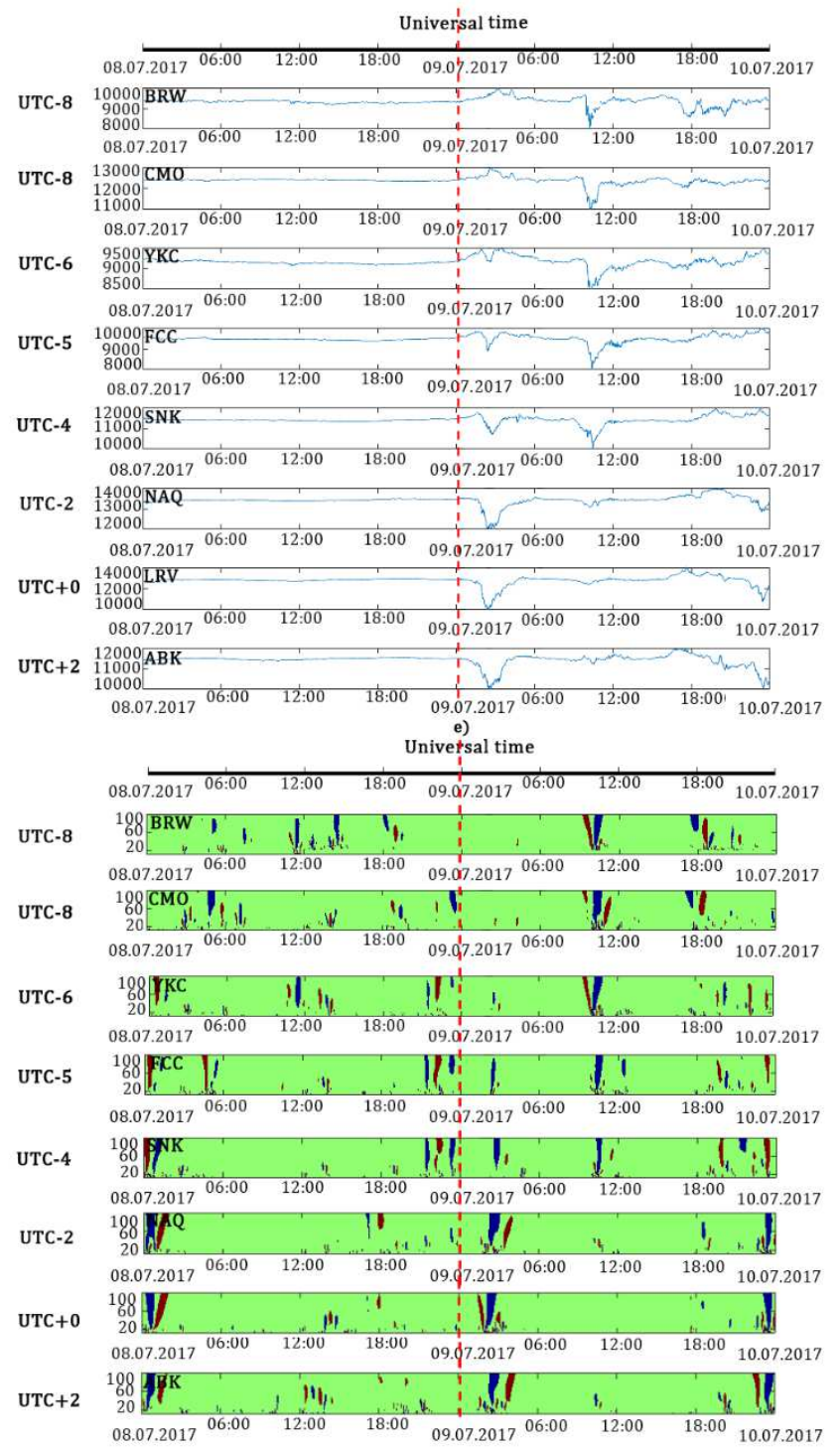

Fig. 5. Results of the auroral zone data processing for the period from 08.07.2017 till 10.07.2017: a) Kp-index; b) Bz-component of interplanetary magnetic field, red color indicates positive values, blue - negative ones; c) auroral indices AE, AO, AL and AU; d) H-component of the magnetic field; e) calculations according to (6), red color indicates positive perturbations, blue - negative ones. The vertical dashed line indicates the magnetic storm onset. 
The second event under analysis occurred on September 27, 2017 (Fig. 6-9). It was caused by a high-speed stream flowing from a large coronal hole (http://spaceweather.com). On the eve of the magnetic storm, the solar wind velocity was $345.7 \mathrm{~km} / \mathrm{s}$, the fluctuations of IMF Bz-components varied within +/- 2 nT. Analysis of the results shows that the rotation periods of IMF Bz-component on September 26 at 06:30 were accompanied by weak increases in the auroral activity indices (Fig. 6a) and short-term perturbations of the geomagnetic field at the analyzed stations (see Fig. 6g,h and 8e,f). The coincidence of periods of increased geomagnetic activity at stations with periods of increasing auroral activity indices, observed during an increase in fluctuations of the Bz-component (Fig. 6c), confirms the relationship of the identified geomagnetic disturbances with nonstationary changes in interplanetary medium parameters.

Note that, similarly with the event considered above on the eve of the storm on September 26, during the period of positive values of IMF Bz-component at auroral stations, an increase in geomagnetic activity is observed (from 09:00 to 12:00 UT at LRV and ABK stations; from 09:00 to 15:00 UT at BRW station (Fig. 9e)). At the same time, at 09:00 UT, synchronous short-term geomagnetic disturbances arise at all meridionally located YAK, PET, and GUA stations (Fig. 8e). A moderate increase in Dst-index was observed during this period from 12:00 to 15:00 UT (Fig. 6e).

At the beginning of the day on September 27, the Bz-component turned southward (at approximately 00:40 UT) and decreased to $-6.7 \mathrm{nT}$. At all analyzed stations during this period, geomagnetic disturbances increased (Fig. 6g,h and 8e,f). At the initial stage of the storm, a moderate increase in Dst-index and auroral activity were observed. The initial phase of the storm was accompanied by a significant increase in geomagnetic activity at middle and high latitude stations (YAK, PET), and a simultaneous increase in auroral activity (Fig. 8a). During the main phase of the storm, geomagnetic field variations at the analyzed stations had different structures (Fig. 8e,f) that is associated with the geographical location of the stations. The rotation periods of IMF southward Bz-component were accompanied by the strongest increases in auroral activity and a significant increase in geomagnetic disturbances at all the analyzed meridian stations. Analysis of auroral zone data (Fig. 7,9) shows the correlation of increases in the indices of auroral activity (Fig. 7c, 9c) and IMF southern component fluctuations (Fig. 7b, 9b) with increasing geomagnetic disturbances at the stations at night (Fig. 7e, 9e). 

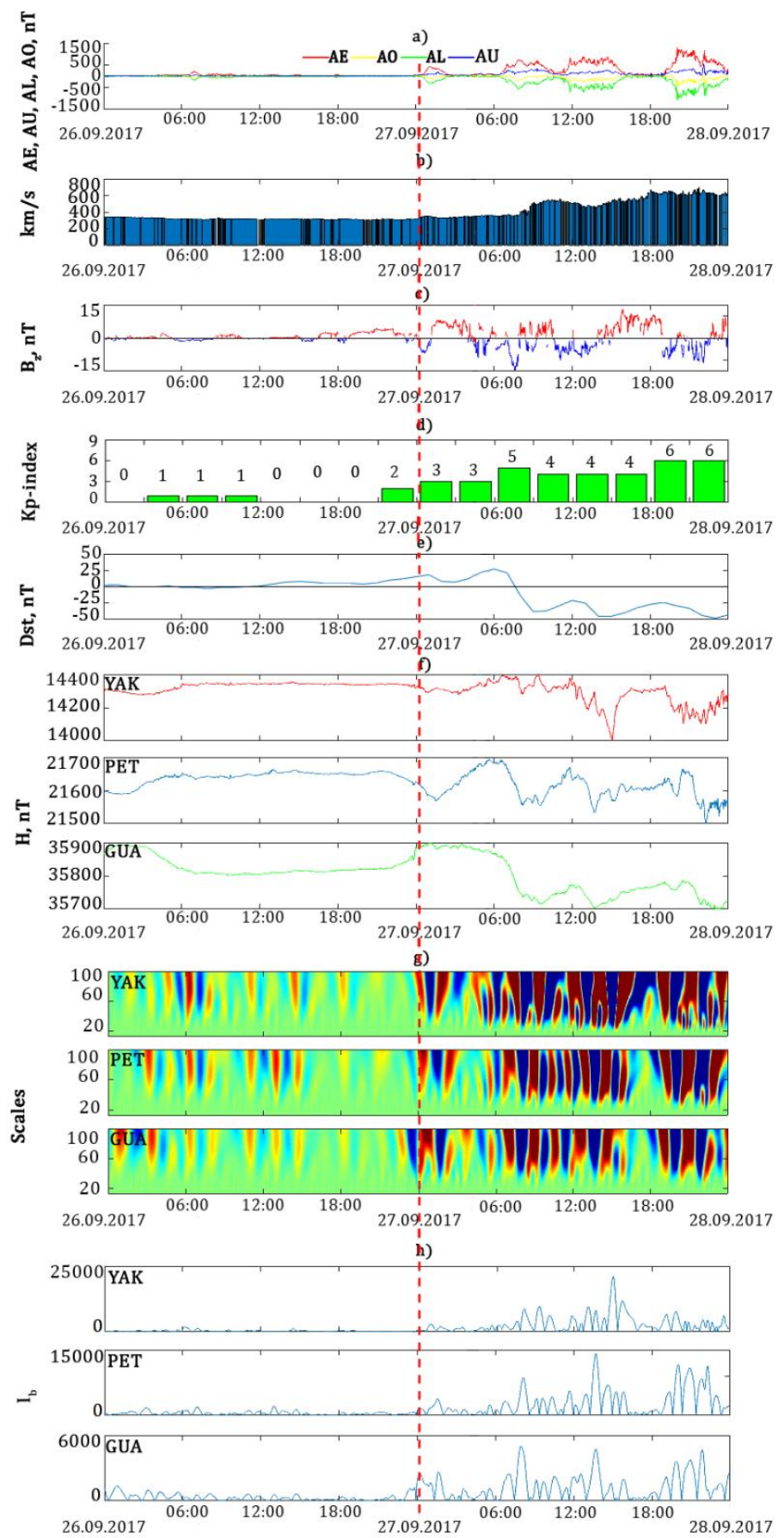

Fig. 6. Data processing results for the period from 26.09.2017 till 28.09.2017:

a) auroral indices $\mathrm{AE}, \mathrm{AO}, \mathrm{AL}$ and $\mathrm{AU}$; b) solar wind speed; c) Bz-component of interplanetary magnetic field, red color indicates positive values, blue - negative; d) Kp-index; e) Dst- index; f) H-component of the magnetic field at PET, YAK and GUA stations; g) wavelet spectrum of geomagnetic disturbances in the regions of PET, YAK and GUA stations (operation (4));

h) absolute values of disturbance intensity in the regions of PET, YAK and GUA stations (operation (5)). The vertical dashed line indicates the magnetic storm onset. 

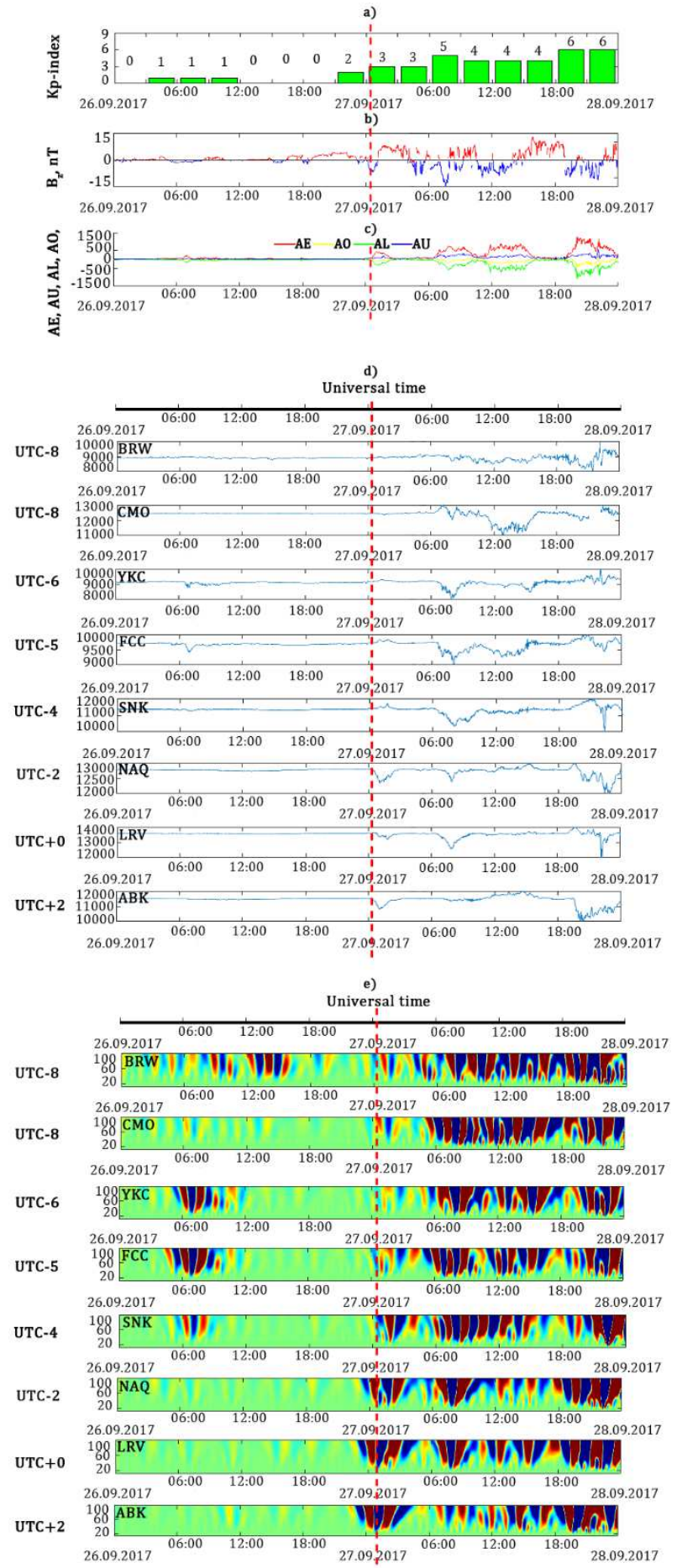

Fig. 7. Results of the auroral zone data processing for the period from 26.09.2017 till 28.09.2017: a) Kp-index; b) Bz-component of interplanetary magnetic field, red color indicates positive values, blue - negative; c) auroral indices $\mathrm{AE}, \mathrm{AO}, \mathrm{AL}$ and $\mathrm{AU}$; d) $\mathrm{H}$-component of the magnetic field; e) wavelet spectrum of geomagnetic disturbances in the regions of the auroral zone stations (operation (4)). The vertical dashed line indicates the magnetic storm onset. 

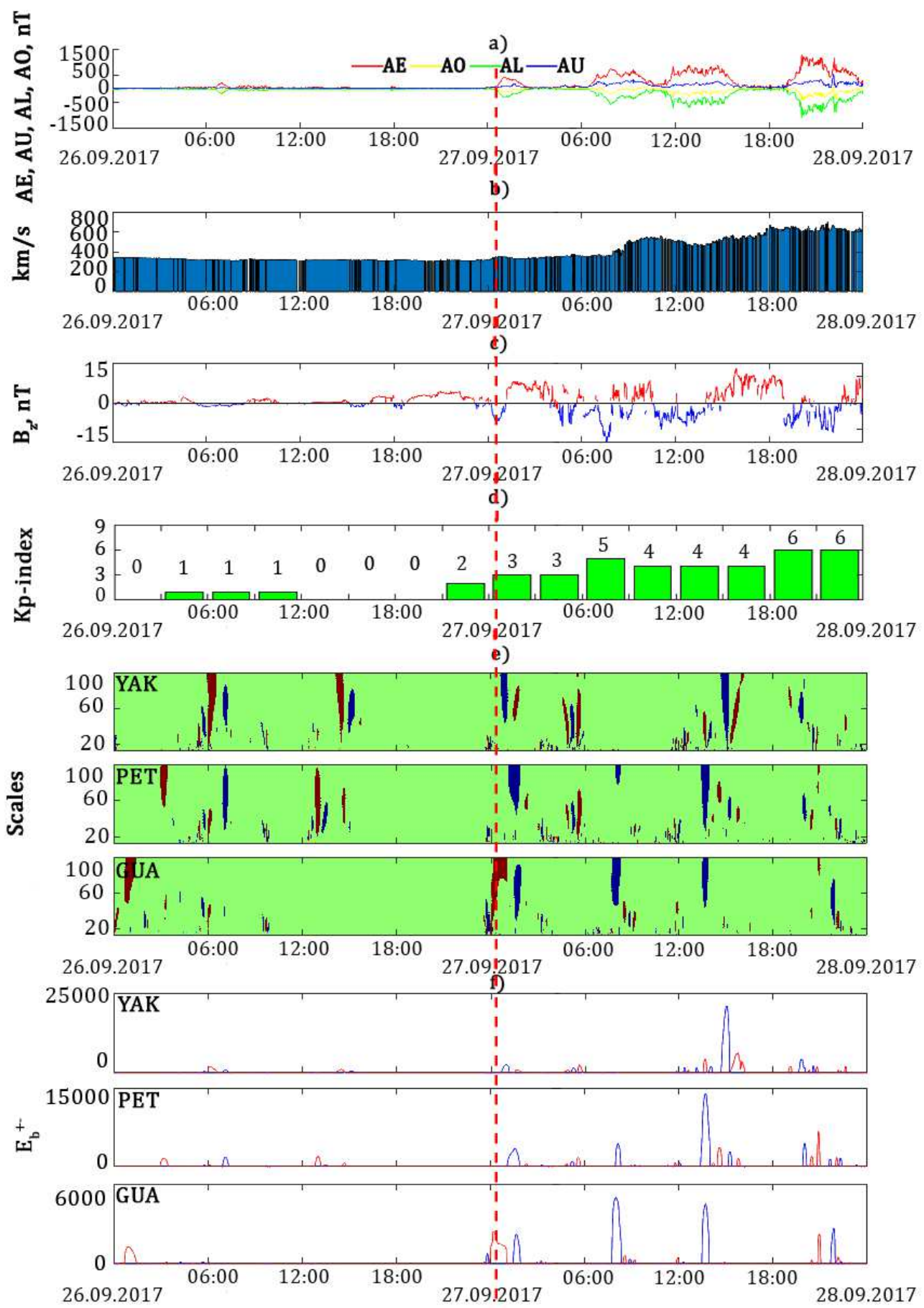

Fig. 8. Data processing results for the period from 26.09.2017 till 28.09.2017:

a) auroral indices $\mathrm{AE}, \mathrm{AO}, \mathrm{AL}$ and $\mathrm{AU}$; b) solar wind speed; c) Bz-component of interplanetary magnetic field, red color indicates positive values, blue - negative; d) Kp-index; e) calculations according to (6), red color indicates positive perturbations, blue - negative ones; f) calculations according to (7), red color indicates positive perturbation, blue - negative ones. The vertical dashed line indicates the magnetic storm onset. 

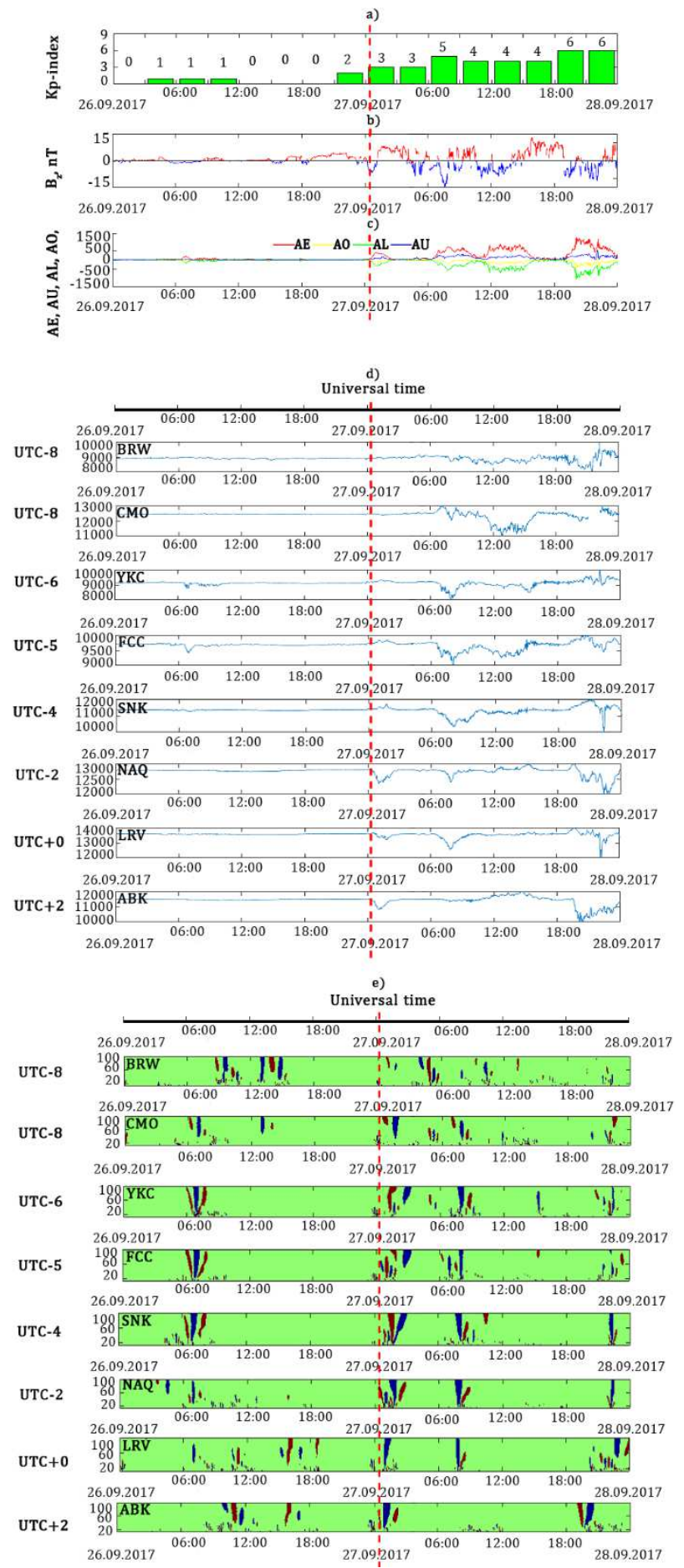

Fig. 9. Results of the auroral zone data processing for the period from 26.09.2017 till 28.09.2017: a) Kp-index; b) Bz-component of interplanetary magnetic field, red color indicates positive values, blue - negative; c) auroral indices $\mathrm{AE}, \mathrm{AO}, \mathrm{AL}$ and $\mathrm{AU}$; d) $\mathrm{H}$-component of the magnetic field; e) calculations according to (6), red color indicates positive perturbations, blue - negative ones. The vertical dashed line indicates the magnetic storm onset. 


\section{Conclusions}

On the basis of the proposed method, a detailed space-time analysis of geomagnetic disturbance dynamics during magnetic storms on July 9, 2017 and September 27, 2017 was carried out. The effectiveness of the method for investigation of unsteady processes in the magnetosphere and for applied researches in the area of space weather forecasting is shown. The method makes it possible to identify weak short-period geomagnetic disturbances preceding magnetic storms that correlate with fluctuations in the interplanetary magnetic field and increases in the auroral indices of geomagnetic activity. We confirmed the assumption made in [10] that short-term increases in geomagnetic activity at equatorial latitudes may occur at the background of small IMF variations and increases in auroral activity.

On the example of the considered events it was revealed that during the periods of IMF Bz-component positive variations, short-term increase of geomagnetic activity is possible both at auroral zone stations and at meridianally located stations (from high latitudes to the equator). The revealed fact confirms the assumption noted in $[6,10]$ that the stability in the magnetosphere is the result of a combination of solar wind and internal magnetospheric activity.

A detailed analysis of the processes in the auroral zone showed the relationship of the identified geomagnetic disturbances with the interplanetary magnetic field parameters and auroral activity indices. The authors plan to continue the research in this direction extending the statistical material and subsequently refining the parameters of the algorithms and increasing their efficiency.

\section{References}

1. N.I. Budko, A.N. Zaitsev, A.T. Karpachev, A.N. Kozlov, B.P. Filippov, Space environment around us (Troitsk: TROVANT, 2006)

2. I. Daubechies, Ten Lectures on Wavelets (CBMS-NSF Lecture Notes, 1992)

3. C.K. Chui, An introduction to wavelets (Academic Press, New York, 1992)

4. A. Jach, P. Kokoszka, J. Sojka, L. Zhu, J. Geophys. Res.111, 1-11 (2006)

5. A. Xu, L. Zhu, J. Sojka, P. Kokoszka, A. Jach, J. Atmospheric Sol.-Terr. 70, 1579$1588(2008)$

6. A.G. Hafez, E. Ghamry, H. Yayama, K. Yumoto, IEEETGRS 50, 4503-4512 (2012)

7. A.V. Vorobiev, Computer optics 41, 963-972 (2017)

8. O.V. Mandrikova, I.S. Solovev, T.L. Zalyaev, EPS 66 (2014)

9. O. Mandrikova, I. Solovev, S. Khomutov, K. Arora, L. Manjula, P. Chandrasekhar, IPERS-ITNT 2017 1901, 180-186 (2017)

10. O.V. Mandrikova, I.S. Solovev, S.Yu. Khomutov, V.V. Geppener, D.M. Klionskiy, M.I. Bogachev, Ann. Geophys. 36 1207-1225 (2018)

11. O.V. Mandrikova, A.I. Rodomanskay, Proceedings of ITNT-2019 4, 140-152 (2019)

12. T. Davis, M. Sugiura, J. Geophys. Res.71, 785-801 (1966)

13. N.A. Zabolotnaya Indices of geomagnetic activity (Moscow: Publisher LKI, 2007)

14. M. Sugiura, Ann. Int. Geophys35, 7-45 (1964)

15. A.N. Zaitsev, V.O. Papitashvili, V.A. Popov Geomagnetism and Aeronomy 26, 156158(1986)

16. X.Y. Zhang, M.B. Moldwin, Space Weather 13, 130-140(2015) 